Dachverband der Technologen/-innen und Analytiker/-innen in der Medizin Deutschland e.V. (DVTA)

\author{
A. M. Gressner ${ }^{1}$ und O. A. Gressner ${ }^{2}$ \\ ${ }^{1}$ Labor Dr. Wisplinghoff Berlin, Berlin, Deutschland \\ ${ }^{2}$ Labor Dr. Wisplinghoff Köln, Köln, Deutschland
}

\section{Synonym(e) DVTA}

Definition Berufsverband, der die Interessen von 4 Berufsgruppen der biomedizinischen Analytik (MTLA, MTRA, MTAF, VMTA) vertritt und deren Aus- und Weiterbildung regelt.

Beschreibung Im April 2016 erfolgten Änderungen von Satzung und Geschäftsordnung, verbunden mit einer Namensänderung, des seit 1969 bis dahin gültigen berufsständischen Verbandes (,Deutscher Verband Technischer Assistenteninnen und Assistenten in der Medizin e.V."), die eine bessere Wahrnehmung der Anliegen und Interessen dieses Berufsverbandes ermöglichen sollen. Im DVTA sind vertreten: Medizinisch-technische Laboratoriumsassistenten/-innen (MTLA), Medizinisch-technische
Radiologieassistenten/-innen (MTRA), Veterinärmedizinischtechnische Assistenten/-innen (VMTA) und Medizinischtechnische Assistenten/-innen der Funktionsdiagnostik (MTAF). Detaillierte Angaben zu Aus- und Fortbildung sowie Weiterqualifikationen (z. B. Bachelor) und Spezialisierungen sind zu erhalten beim Deutschen Institut zur Weiterbildung für Technologen/-innen und Analytiker/-innen in der Medizin e.V. (DIW-MTA, Welserstr. 5-7, D-10777 Berlin, Tel. 030-33844064).

\section{Adresse:}

Dachverband für Technologen/-innen und Analytiker/innen in der Medizin Deutschland e.V.

Geschäftsstelle

Spaldingstr. 110b

D-20079 Hamburg

Tel.: 040-2351170

Fax: 040-233373

E-Mail: info@dvta.de

\section{Literatur}

MTA-Dialog, Fachzeitschrift für medizinisch-technische Assistenten, www.mta-dialog.de 